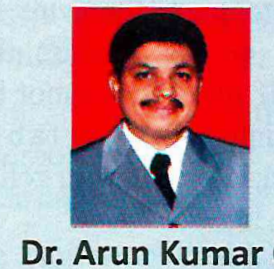

Professor

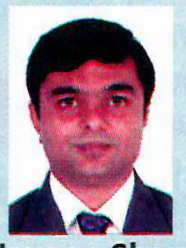

Dr. Naveen Shamnur

Professor

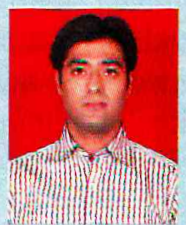

Dr. Siddharth Brijwani

Post graduate student

Department of Orthodontics \& Dentofacial Orthopaedics

\title{
Introduction
}

True orthodontic emergencies are very rare, but when they do occur, require immediate attention. As a general rule patients should report/call to the clinics when they experience severe pain or when they encounter some painful appliance problems that cannot be taken care by them.

It may come as a surprise that many of the problems can be solved temporarily by the patients themselves, if they are well acquainted with their appliances. About various parts of the appliances and their functions and ease of identification should be told to the patients beforehand to avoid any panic during the time of an emergency.

Let us look at some materials we use on regular basis

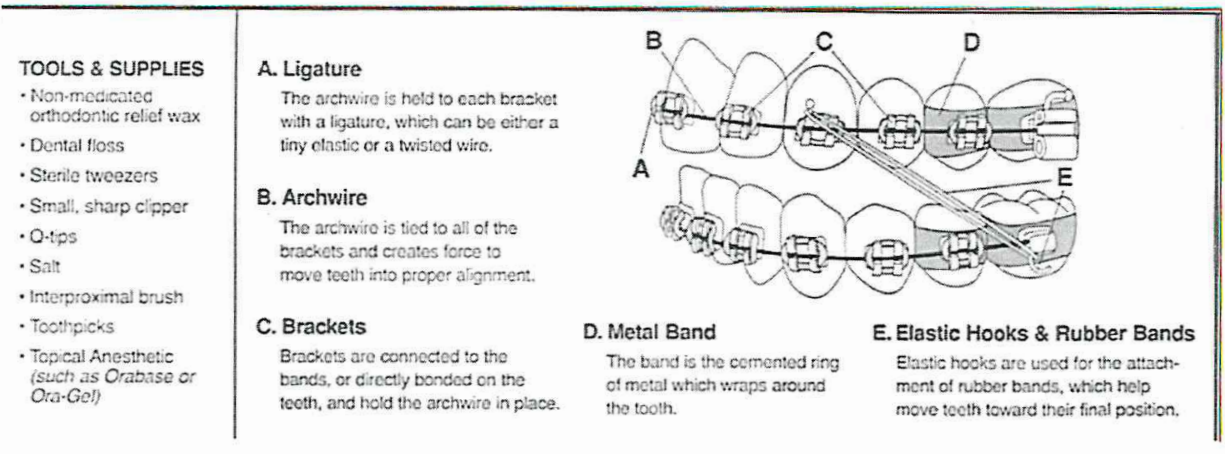

\section{Emergencies And Their Remedies}

The following orthodontic emergencies and their treatments are listed in the order of the least severe to the most severe. Only the most severe emergencies may require immediate attention by an orthodontist. The majority of these are easily treated with a follow-up by the patient's orthodontist.

\section{1) Food Caught Between Teeth}

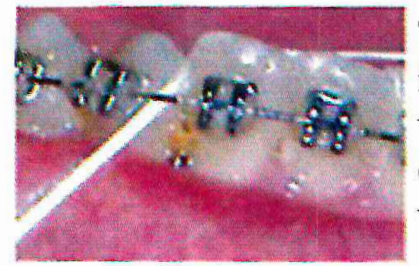

This is not an emergency, but it can be a little uncomfortable or embarrassing for the braceswearing patient.

Management:

It can be easily removed with a piece of dental floss. By tying a small knot in the middle of the floss to help remove the food

use an interproximal brush or toothpick to dislodge food caught between teeth and braces.

\section{2) Ligatures Come Off}

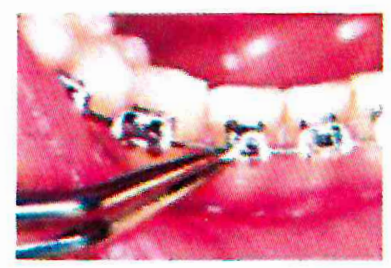

Tiny rubber bands or small, fine wires, known as ligatures, hold the wire to the bracket.

If a rubber ligature have come off one may be able to put it back in place using sterile tweezers.

If a wire ligature comes loose, simply remove it with sterile tweezers.

If the wire ligature is slicking out into the lip but is not loose, it may be bent back down with a Q-tip or pencil eraser to eliminate the irritation.

In case, when one ligature pops off or breaks, others may follow. Be sure to examine all ligatures. Missing or broken ligatures should be brought to the attention of the patient's parent/ guardian, who should then inform the orthodontist.

If a rubber or wire ligature is lost, notify the parent/ guardian so that the orthodontist may advise whether the patient should be seen or not. 


\section{3) Discomfort/Soreness}

-It's normal for a patient to have discomfort/sore teeth for a day or two after braces or retainers are adjusted which can make eating uncomfortable.Some patients are susceptible to episodes of mouth sores. While braces do not cause them, they may be precipitated or exacerbated by an irritation from braces. This is not an emergency, but may be very uncomfortable for the patient.

- Reassure the patient that the discomfort is both normal and temporary

- Encourage soft foods.

- Have the patient rinse the mouth with warm salt water.Dissolve one teaspoonful of salt in eight ounces of warm water, and rinse your mouth vigorously.

- Prompt relief may be achieved by applying a small amount of topical anesthetic directly to the ulcerated surface using a cotton swab. Instruct $\mathrm{t} \mathrm{h}$ e patient to reapply as required.

- If the tenderness is severe, Paracetamol can help to relieve this discomfort.

\section{4) Irritation of Lips or Cheeks}

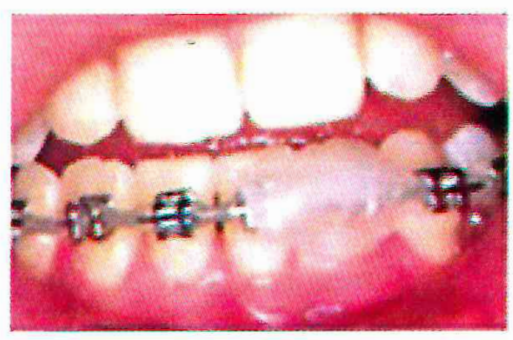

-Sometimes new braces can be irritating to the mouth, especially when the patient is eating. Occasionally patients may develop canker sores while wearing braces.Canker sores generally heal within $7-10$ days. There is no cure for canker sores, but to help relieve any discomfort we recommend placing wax on the problematic area of the braces and use any anesthetic gel base on the sore spot. Let the patient know that if the wax is accidentally ingested, it's not a problem. The wax is harmless.

- Simply pinch off a small piece of wax and roll it into a ball the size of a small pea.

- Flatten the ball and place it completely over the area of the braces causing irritation.

- One or several areas of ulceration of the cheeks, lips or tongue may appear.

\section{5) Protruding Wire}

Occasionally, the end of a wire will work itself out of place and irritate the patient's mouth.

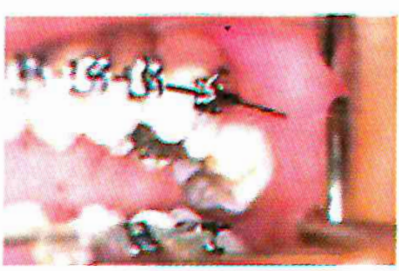

- Use a Q-tip or pencil eraser to push the wire so that it is flat against the tooth. If the wire cannot be moved into a comfortable position, cover it with relief wax.

- Alternatively a chewing gum/cotton roll can also be used if wax is unavailable.

- The patient's parent/guardian will need to make the orthodontist aware of the problem.

- In a situation where the wire is extremely bothersome and the patient will not be able to see the orthodontist anytime soon, you may as a last resort clip the wire using sharp clippers.

- Reduce the possibility of the patient swallowing the snipped piece of wire by using folded tissue or gauze around the area.

\section{6) Loose Brackets, Wires or Bands}

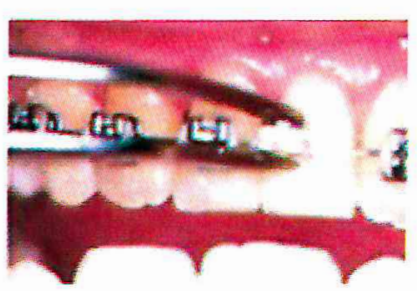

-Brackets are the parts of braces attached to teeth with a special adhesive. They are generally positioned in the center of each tooth. The bracket can be knocked off if the patient has eaten one of those hard or crunchy foods, orthodontic patients are instructed to avoid, or if the mouth is struck while at play.

- If the braces have come loose in any way. Theparent/guardian needs to be notified, and they should call the orthodontist to determine the appropriate next steps.

- Encourage all patients, especially those with braces, to wear a protective mouth guard while playing sports.

- If the bracket is off center, the adhesive may have failed. Call the parent/guardian, and recommend that they immediately notify the orthodontist, who will determine the course of action.

- If the loose bracket has rotated on the wire and is sticking out and the patient cannot immediately be taken to the orthodontist, you can do a temporary fix to alleviate discomfort and prevent further damage. But take care to prevent swallowing or other injury.

- To put the bracket back in place, use sterile tweezers to slide the bracket along the wire until it is between two teeth. Rotate the bracket back to the proper position, and then slide it back to the center of the tooth. 


\section{7) Piece of Appliance is Swallowed}

This is rare, but when it does happen, it can be fairly alarming to the patient.

If you are able to see the piece, you may carefully attempt to remove it. But do not make the attempt if you could cause harm.

The aspiration of a foreign body during dental treatment presents a serious problem, and the symptoms depend on where the object becomes impacted.

If it is trapped above the vocal cords, acute respiratory distress can result; this requires urgent action.

Smaller objects, however, tend to pass through the vocal cords, and upper airway obstruction does not occur.

The commonest symptoms of laryngotracheal foreign bodies are dyspnea, Cough and stridor; those of bronchial foreign bodies are cough, decreased air entry, dyspnea, and wheezing.

- Management of aspirated foreign bodies depends on the severity of the symptoms.

- Encourage your patient to remain calm. If the patient is coughing excessively or having difficulty breathing, the piece could have been aspirated.

- If the foreign body is obstructive and the patient is in respiratory distress, dislodgement of the foreign body should be initially attempted with back blows and the Heimlich maneuver.

- If these fail to dislodge the object, positive airway pressure needs to be maintained by artificial respiration;

- If this fails to maintain a patent airway, the object should be bypassed, and an emergency airway established.

\section{Clinical recommendations:}

Appliances or their components can become dislodged if retention is inadequate, and potentially serious complications to the larynx, pharynx, or gastrointestinal tract can arise. Problems have been caused by a variety of appliances, and the following precautions are recommended:

1. When adjusting small components intraorally, place a gauze/ dental napkin behind the orthodontic appliance to act as a barrier.

2. Tie floss leashes onto expansion keys, transpalatal arches, quad-helices, molar bands, and any other loose components when adjusting them intraorally. Keys attached to a plastic ring holder are commercially available.

3. Use wax to temporarily stabilize auxiliaries, such as coil springs, on archwires.
4. Use acrylics in colors other than pink to construct removable appliances and retainers. Removable appliances should be suitably retentive and of an adequate size. Avoid sharp edges and hooked or pointed wire components. Check the appliances regularly.

5. Consider radiopaque acrylic for clear orthodontic appliances; however, these materials might possess compromised strength and esthetics.

6. Advise patients both verbally and with written instructions during appliance placement that they should not try to reinsert damaged, ill-fitting, or broken fragments of any appliance. They should stop using them immediately until the appliance is checked.

7. Select archwire material and grade not only for the forces required to move teeth, but also for the wireability to withstand masticatory stresses.

8. Before cutting the ends of archwires intraorally with safety distal-end wire cutters, place a cotton roll over the end of the archwire.

9. Regularly inspect all orthodontic instruments for signs of failure; replace or recondition these instruments on a regular basis.

10. When taking impressions, use a material with high viscosity and trays that fit properly. An upright position for the patient is recommended with clear instructions. The clinician should be able to visualize the back of the upper tray as it is seated in the mouth so that excess impression material does not extrude into the back of the mouth. The patient should then be advised to tilt the head forward, chin down, and avoid swallowing.

11. If a piece of appliance is dropped in the mouth during treatment, high-speed suction with a pharyngeal tip can help with quick retrieval.

12. In cases of respiratory distress resulting from aspiration of an object, the Heimlich maneuver is recommended. If the patient is in severe distress, maintain positive air pressure with cardiopulmonary resuscitation until medical help arrives. Keep up to date with cardiopulmonary resuscitation, since the recommendations can change.

13. Immediate attention and elective intervention as suggested by medical consultants should be done to prevent the risk of perforation.

8) Accidents involving teeth

In case of broken teeth or any type of RTA, patient should immediately report to the clinics or to the dentist. If patients are involved in contact sports, a regular mouth guard can be fitted over braces for added protection. 
Communication through telemedicine: home teleassistance in orthodontics

Telecommunications applied to medicine and dentistry is currently a subject of topical interest. The most developed countries are investing resources in order to improve assistance and communication between physicians and patients and among specialists. The possibility of sharing videos and images is particularly useful in the orthodontic field, as minor emergencies can be solved easily at home, reassuring patient and parents on one hand, and limiting visits to the dental office to cases of real need.

References:

1. Dibiase AT, Samuels RH, Ozdiler E, Akcam MO, Turkkahraman H.Hazards of orthodontics appliances and the oropharynx. J Orthod2000;27:295-302.

2. Abdel-Kader HM. Broken orthodontic trans-palatal archwire stuckto the throat of orthodontic patient: is it strange? J Orthod 2003;30:11.

3. Cameron SM, Whitlock WL, Tabor MS. Foreign body aspiration indentistry: a review. J Am DentAssoc 1996;127:1224-9.

4. Milton TM, Hearing SD, Ireland AJ. Ingested foreign bodies associated with orthodontic treatment: report of three cases and review of ingestion/aspiration incident management. Br Dent J 2001;190:592-6.

5. Alaluusua S. Emergency therapy in orthodontics.Arsb Odontol SamfFinl. 1980:5-6

6. Wickwire NA, Norton LA. Treatment of orthodontic emergencies in general practice.ASDC J Dent Child. 1972 JanFeb;39(1):23-8.

7. Gottlieb EL.Editorial. Most orthodontic emergencies are not emergencies at all.JPO J Pract Orthod. 1969 Jul;3(7):335-6.

8. Bilder L, Hazan-Molina H, Aizenbud D.Medical emergencies in a dental office: inhalation and ingestion of orthodontic objects. JAm DentAssoc. 2011 Jan;142(1):45-52.

9. Favero L, Pavan L, Arreghini A. Communication through telemedicine: home teleassistance in orthodontics.Eur J Paediatr Dent. 2009 Dec;10(4):163-7.

\section{NATIONAL AWARDS WON BY IDA-DAVANGERE BRANCH AT $65^{\text {TH }}$ IDA NATIONAL CONFERENCE HELD AT MUMBAI}

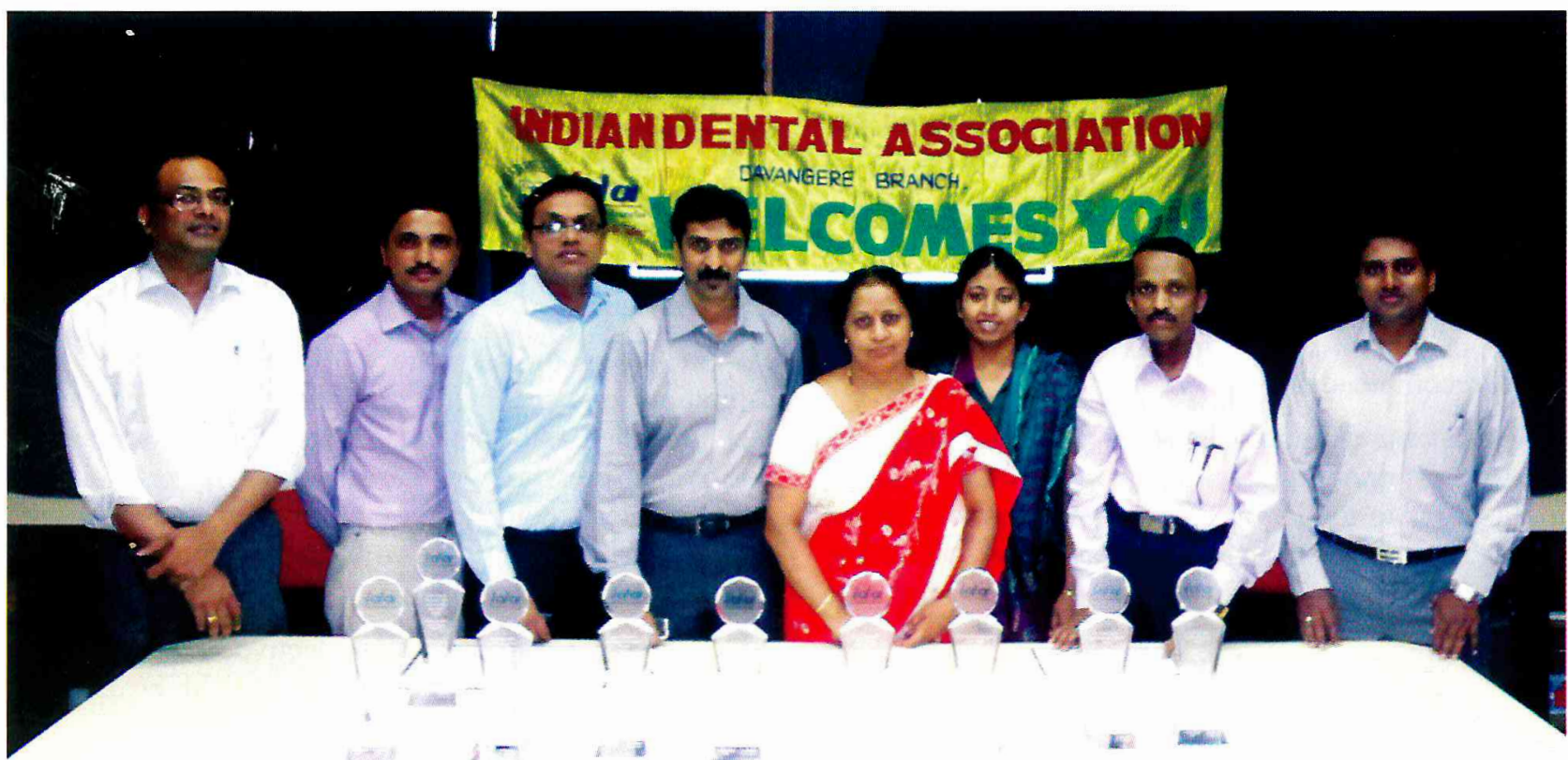

- Best local branch

- Best local branch president

- Best local branch journal (Runner up)

- Best local branch Secretary

- Best membership drive
- Best CDE Chairman

- Best CDH Chairman

- Best student activity

- Best all round activity

- Best school dental health program activities 